\title{
Liquid Chromatography: Introduction and Instrumentation
}

\author{
Lane C. Sander \\ National Institute of Standards and Technology, \\ Gaithersburg, MD 20899, USA \\ lane.sander@nist.gov
}

Video DOI: http://doi.org/10.18434/T4DK5T

Key words: check valves; chromatographic pumps; instrumentation; LC theory; liquid chromatography; operation.

Accepted: December 1, 2016

Published: January 12, 2017

https://doi.org/10.6028/jres.122.001

\section{Summary}

Chromatography is an example of a process by which a mixture is separated into at least two fractions with different compositions. This presentation will provide a brief overview of the practice of liquid chromatography (LC), with an emphasis on chromatographic instrumentation. Instrumentation often differs significantly among manufacturers, but the principles of operation are the same. Analytical methods usually can be transferred from one instrument to another, as long as the basic requirements are maintained. Some variability is to be expected based on differences in instrumentation, but the changes in performance can often be compensated by adjusting operational parameters.

The presentation includes a brief historical perspective and schematic illustration of column chromatography. The design and operation of modern chromatographic instrumentation is described, including individual components. The principle of operation of a reciprocating piston pump with inlet and outlet check valves is illustrated by an animated sequence. An overview of LC detectors is provided to include absorbance detectors (single wavelength, variable wavelength, and diode array), and detectors based on fluorescence, refractive index, evaporative light scattering, electrochemical, or mass spectrometry. A laboratory video is included to demonstrate the startup procedure and operation for a typical liquid chromatograph. ${ }^{1}$

\footnotetext{
${ }^{1}$ Contribution of the National Institute of Standards and Technology. Not subject to copyright. Certain commercial equipment, instruments, or materials are identified to specify adequately the experimental procedure. Such identification does not imply recommendation or endorsement by the National Institute of Standards and Technology, nor does it imply that the materials or equipment identified are the best available for the purpose.
} 